\title{
BMJ Open Strategy to reduce adverse health outcomes in subjects highly vulnerable to COVID-19: results from a population- based study in Northern Italy
}

\author{
Antonio Giampiero Russo (D) , ${ }^{1}$ Marino Faccini, ${ }^{2}$ Walter Bergamaschi, ${ }^{3}$ \\ Antonio Riussi ${ }^{1}$
}

To cite: Russo AG, Faccini M, Bergamaschi W, et al. Strategy to reduce adverse health outcomes in subjects highly vulnerable to COVID-19: results from a population-based study in Northern Italy. BMJ Open 2021;11:e046044. doi:10.1136/ bmjopen-2020-046044

- Prepublication history for this paper is available online. To view these files, please visit the journal online (http://dx.doi. org/10.1136/bmjopen-2020046044).

Received 19 0ctober 2020 Revised 16 January 2021 Accepted 22 February 2021

Check for updates

(C) Author(s) (or their employer(s)) 2021. Re-use permitted under CC BY-NC. No commercial re-use. See rights and permissions. Published by BMJ.

${ }^{1}$ Epidemiology Unit, Agency for Health Protection of the Metropolitan Area of Milan, Milano, Italy

${ }^{2}$ Infectious Diseases Unit, Prevention Department, Agency for Health Protection of the Metropolitan Area of Milan, Milano, Italy

${ }^{3}$ General Directorate, Agency for Health Protection of the Metropolitan Area of Milan, Milano, Italy

Correspondence to Dr Antonio Giampiero Russo; agrusso@ats-milano.it

\section{ABSTRACT}

Objectives This study describes a new strategy to reduce the impact of COVID-19 on the elderly and other clinically vulnerable subjects, where general practitioners (GPs) play an active role in managing high-risk patients, reducing adverse health outcomes.

Design Retrospective cohort study.

Setting Population-based study including subjects resident in the province of Milan and Lodi.

Participants 127735 residents older than 70 years, with specific chronic conditions.

Interventions We developed a predictive algorithm for overall mortality risk based on demographic and clinical characteristics. All residents older than 70 years were classified as being at low or high risk of death from COVID-19 infection according to the algorithm. The highrisk group was assigned to their GPs for telephone triage and consultation. The high-risk cohort was divided into two groups based on GP intervention: patients who were not contacted and patients who were contacted by their GPs. Outcome measures Overall mortality, COVID-19 morbidity and hospitalisation.

Results Patients with increased risk of death from COVID-19 were 127 735; 495669 patients were not at high risk and were not included in the intervention. Out of the high-risk subjects, 79110 were included but not contacted by their GPs, while 48625 high-risk subjects were included and contacted. Overall mortality, morbidity and hospitalisation was higher in high-risk patients compared with low-risk populations. High-risk patients contacted by their GPs had a $50 \%$ risk reduction in COVID-19 mortality, and a $70 \%$ risk reduction in morbidity and hospitalisation for COVID-19 compared with noncontacted patients.

Conclusions The study showed that, during the COVID-19 outbreak, involvement of GPs and changes in care management of high-risk groups produced a significant reduction in all adverse health outcomes.

\section{INTRODUCTION}

On 19 February 2020, the first case of COVID-19 was identified in Italy, in the province of Milan. Previously, the epidemic had been confined to China, with several restrictions active at the national and international
Strengths and limitations of this study

- This is the first population-based study that includes all people resident in the largest metropolitan area of Northern Italy and focuses on patients at high-risk for COVID-19 mortality.

- We defined a highly discriminating algorithm model, based on administrative health data, to identify groups experiencing high mortality.

- The study highlights how the involvement of general practitioners and implementation of changes in the care management of high-risk groups produce a significant reduction in all adverse health outcomes.

- The multiprofessional intervention involved patients, general practitioners, epidemiologists, IT experts and politicians and entailed a rapid data collection during a rapidly evolving pandemic emergency.

- As this pragmatic study in a COVID-19 hot spot is analysed retrospectively and not randomised, it does not guarantee protection from residual confounding due to unmeasured variables.

levels. Since large Chinese communities are present in many Italian metropolitan areas, the first cases were expected in these areas. Instead, the first case occurred in a nonmetropolitan area, in the municipality of Codogno, which has just over 15000 inhabitants. The first case had an initial non-specific set of symptoms that delayed the identification of COVID-19. After the first case, 46 close contacts, including healthcare staff of the Codogno hospital, were infected, effectively starting the Italian phase of the COVID-19 pandemic. $^{1}$

On 23 February, 50000 residents were ordered into isolation in the municipalities surrounding Codogno; despite this, COVID-19 spread to the provinces of Cremona, Mantua, Bergamo and Brescia. Pursuing the same strategy as the Chinese government, a national lockdown was ordered on 8 March. $^{23}$ 
Without effective prevention or treatment measures, the main strategy to control COVID-19 focused on different community-based intervention models. To address virus transmission, governments worldwide faced a choice between mitigation or suppression strategies. ${ }^{45}$

Some governments opted for suppression, as more than 40 countries or regions worldwide enacted population lockdowns. ${ }^{67}$ Other governments aimed for mitigation, trying to slow rather than stop the spread of the virus. ${ }^{8}$

Each country developed specific approaches based on mixing surveillance and testing strategies, community and distancing measures and healthcare reforms to increase intensive care capacity and reduce all other non-essential medical activities. ${ }^{9}$ Although less than $1 \%$ of patients with COVID-19 die, the sheer number of infections resulted in increased hospital admissions, and most patients needed intensive care. Consequently, hospitals became overburdened, and deaths increased. ${ }^{10}$

The majority of patients hospitalised with COVID-19 were older and had underlying medical conditions, with increased age being associated with clinical severity, including case fatality. The most frequent comorbidities reported in Chinese patients with COVID-19 were hypertension $(20 \%)$, diabetes $(10 \%)$, cardiovascular disease $(8 \%)$ and respiratory system disease $(2 \%) .{ }^{11} 12$

In the US population, common comorbidities were hypertension (60\%), obesity (40\%) and diabetes $(34 \%) .{ }^{13} 14$

On 1 March, with data released at the regional level providing a clear idea of the heavy human, social and economic losses, we observed the progress of the epidemic without any tool other than our knowledge as public health epidemiologists. Was it possible that epidemiologists, during this pandemic event, could only observe the progress of events and could take no other action based on their experience?

The world stopped, cities were deserted and people were locked in their homes. Under widespread lockdown conditions, we studied a rapid, although rudimentary, predictive model to identify the characteristics of the elderly population dying from COVID-19. Our main idea was to generate a rapid, simple information system capable of incorporating the cohort of subjects more susceptible to dying into a web application that would allow general practitioners (GPs) to operate actively, in particular since clinics were closed due to the restrictive measures implemented. With an extensive effort, even considering the extremely short time available due to the speed of contagion, this system provided GPs of Milan's Agency for Health Protection (Agenzia per la Tutela della Salute (ATS) ) with a tool to protect, treat and supervise those who needed it most at that time. All that was needed was a staunch resolution to transform an idea into a real action plan in the midst of a public health emergency; this could be done only by the general manager of the ATS.

The vivid portrayal previously is necessary to introduce the concept that most COVID-19 patients are asymptomatic, and infection becomes clinically relevant most frequently in elderly patients with comorbidities. The national strategic plan did not specify, at the population level, all surveillance actions needed to trace the most at-risk patients and include them in a system to limit adverse health and social effects; only general recommendations were issued instead.

This work aims to present the results of a populationbased intervention on the elderly and COVID-19 vulnerable population. The intervention used a mortality prediction model based on early data that implements a web-based information system and a concerted effort by GPs. This multidisciplinary action, coordinated by the Epidemiology Unit of Milan's ATS and performed by GPs in the provinces of Lodi and Milan, represents a new consensus model to reduce mortality rates during public health emergencies.

\section{MATERIALS AND METHODS Information system}

From the beginning of the outbreak, all tracing activities were included in a newly dedicated web-based platform called Milano $\mathrm{COV}$, which allows for identification of cases and related contacts. The ATS health system contains data on all laboratory-confirmed cases of COVID-19. Confirmation of COVID-19 infection was provided by reverse transcription PCR (RT-PCR) on nasopharyngeal swabs performed in 12 regional laboratories.

The system includes only laboratory-confirmed cases of COVID-19 infection, irrespective of clinical symptoms. Contacts are defined as all individuals associated with some range of activity of the case and who may have similar exposures as the case. Contacts can include household members, other family members, visitors, neighbours, colleagues, teachers, classmates, coworkers, social or health workers and members of social groups. The epidemiological investigation among cases and contacts was set to provide descriptions of the clinical presentation of COVID-19 infection and course of associated diseases, serial interval of COVID-19 infection, symptomatic proportion of COVID-19 cases and identification of possible transmission routes.

In the first week of March, the web portal was available to all GPs, allowing them to identify cases and close contacts and conduct telephone follow-up with non-hospitalised cases. It also allowed GPs to communicate with any symptomatic cases and their close contacts asking for therapeutic indications. Furthermore, at the time, GPs also gave indication that quarantine should be maintained as if they were confirmed cases/close contacts.

During the outbreak, mayors were to be sent all data relating to identified cases and contacts. A list of cases and close contacts was provided, for each municipality, in order to ensure that containment measures were followed and verify the need for any social support (eg, grocery shopping and medications). The prefectures in Milan and Lodi used the same information to enforce the 
restrictive measures provided for by law. The same informational structure also allowed for tracing case evolution and follow-up of cases until PCR negativisation and the end of quarantine for close contacts. Additionally, GPs had the option of adding exclusively symptomatic cases and their close contacts to expand the outbreak reporting system.

This integrated platform (ATS Milano COR) contained personal data and detailed information on both cases and contacts, identified through epidemiological investigations, and GP-reported symptomatic cases and contacts, available to 193 municipal administrations, for specific population interventions, and to the prefectures of Milan and Lodi, for all containment and support measures provided for by specific legislation.

For cases and contacts reported by GPs, in addition to starting surveillance based on active tracing in the general population, massive SMS systems were activated both to strengthen the indications for segregation and send links with information on how to quarantine at home.

\section{Patient and public involvement}

Patients who took part in the GP intervention were involved in the study, as they were contacted by telephone and received specific indications from their GPs. Results will be presented to the GPs of Milan's ATS after formal acceptance and publishing by a peer-reviewed journal.

\section{Predictive algorithm for overall mortality risk}

In the first fortnight of March, available data were used to develop a predictive algorithm for overall mortality risk. Predictors were chosen based on the magnitude of the association and reproducibility of the results that emerged each day (not for significance), as well as clinical relevance, by using logistic regression analysis. The final models predicting mortality risk in COVID-19 cases included neurological disorders (dementia, Alzheimer's disease and Parkinson's disease), heart failure, ischaemic cardiomyopathy, valvular disease, renal failure (including dialysis) and cancer diagnosed in the last 2 years.

\section{Classification of high-risk subjects}

Only patients over the age of 70 years and under the care of GPs of Milan's ATS were included. All residents of nursing homes, extended care units and long-term care facilities were excluded. All subjects classified as at high risk of death were included in the prospective intervention. Patients in the cohort were distributed based on the GP in charge of their care by using ATS Milano COR. The platform allowed secure access to every GP who contacted patients by telephone. In addition to ascertaining clinical conditions, reviewing treatment and highlighting any medical problems, GPs explained the risks of infection in already compromised patients, reiterated the need for isolation and determined whether there was any need for social services. If the patient did require social services, a timely request was sent to the appropriate social service organisations in the municipality of residence. ATS Milano
COR informed each GP if there was any conversion of a high-risk chronic case into a confirmed case and if the patient had been hospitalised.

\section{Outcome variables}

Overall mortality

Vital status was derived from the early notification system of the ATS of Milan, set up at the beginning of the epidemic, in which deaths are communicated from the civil registry of each municipality to the ATS, manually entered in the Health Service Register, and directly from the GPs' and mayors' offices for patients already in the COVID-19 database through the web-based information system.

\section{COVID-19 morbidity}

Morbidity rates of confirmed COVID-19 cases from the high-risk cohort were identified using the registry of molecular RT-PCR diagnostic testing results based on nasal swabs.

\section{COVID-19 hospitalisation}

Hospitalised confirmed COVID-19 cases were identified using a specific regional flow. For all confirmed cases, the information system provides the date and location of hospitalisation and the date of discharge.

\section{Statistical analysis}

To evaluate the beneficial effect of the developed classification, the population of patients aged over 70 years was stratified into patients not included in high-risk surveillance (G1), included but not contacted by GPs (G2) and included and contacted by GPs (G3).

Mortality, morbidity and hospitalisation events were identified from the date on which the system started up (13 March) to the date on which mortality of the general population went back to its pre-epidemic expected value (31 May). All events after the end of the study observation period were censored and considered non-events.

Both multivariate logistic analysis and proportional hazards models were used to estimate ORs and HRs, and 95\% CIs were used to assess the relationships between mortality, morbidity and hospitalisation.

Separate models were fitted to the data comparing G2+G3 together versus G1, G2 versus G1 and G3 versus G1, and, finally, G3 versus G2. Individual-level comorbidities data were derived using the administrative chronic disease database of the ATS of Milan, according to the algorithms specified in Regional Act X/616418 and $\mathrm{X} / 765519$ of 2017 . The number of different pharmacological treatments was defined on the basis of the sevendigit ATC code and subdivided into a polytherapy group (more than five treatments but less than 10) and a hypertherapy group (more than 10 tracers), using those who used less than five different treatments as a reference.

Overall systemic health was determined using the Charlson Comorbidity Index (CCI) score, which assigns patients a score between 0 and 6 , based on the likelihood of mortality secondary to age and the presence or 
absence of selected systemic diseases. The CCI includes myocardial infarction, congestive heart failure, peripheral vascular disease, cerebrovascular disease, dementia, chronic pulmonary disease, rheumatologic disease, peptic ulcer disease, cirrhosis, hepatic failure, immunosuppression, diabetes mellitus with or without complications, haemiplegia or paraplegia, chronic renal disease, malignant neoplasms, multiple myeloma or leukaemia, lymphomas, metastatic solid tumours and AIDS. ${ }^{15}$

For logistic regression analysis, the model prediction was calculated using Harrell's C-statistic: up to 0.5 (as good as a random guess), 0.7 to 0.8 (acceptable), 0.8 to 0.9 (excellent), and 0.9 to 1.0 (outstanding prediction). ${ }^{16}$

The Cox proportional hazard assumption was checked for each covariate using Schoenfeld residuals tests and graphically using a log-log plot of survival.

All statistical analyses were performed using SAS V9.4 for Windows (SAS Institute, Inc, Cary, North Carolina, USA).

\section{RESULTS}

\section{Predictive algorithm for overall mortality risk}

On 13 March, there were 2981 confirmed cases of COVID19. Of these, 435 (14\%) died before 15 March 2020. A total of 1004 cases were 70 years and older, and of these, $392(28 \%)$ had died. On the date the prediction model was implemented, the C-index was 0.81 .

\section{Cohorts}

On 20 April 2020, 623404 patients aged over 70 years were identified. Of these, 127735 (20.5\%) had comorbid conditions that increased their risk of death from COVID19. At the time of cohort inclusion, there were 2047 GPs in the ATS of Milan; 1454 (71\%) logged on to ATS Milano COR and 1132 (55\%) contacted more than five patients via telephone (min-max: 5-153, mean: 43, median: 41), for a total of 48624 patients (38\%). The mean age of patients contacted by GPs was 59 versus 61 for patients not contacted; $59 \%$ of complying GPs worked in the city of Milan, while $48 \%$ worked in another province of Milan and Lodi. Sociodemographic characteristics of the patients belonging to the two groups of GPs were not different: female gender ( $51 \%$ not contacted vs $53 \%$ contacted), patients older than 70 years (19\% vs $19 \%$ ) and foreigners (14\% vs 14\%).

By using all the clinical conditions included in the algorithm, 127735 patients out of 623404 (20.5\%) over the age of 70 years were classified as at high mortality risk for COVID-19. The cohort of patients older than 70 years was subdivided into three different cohorts: (G1) without any specific risk conditions (495 669; 79\%); (G2) with risk conditions but not contacted by GPs (79 110; 13\%); and (G3) with risk conditions in contact with GPs (48 625; $8 \%$ ). Table 1 shows the baseline characteristics of the different cohorts: included and contacted patients were very close to non-contacted patients in age and gender distribution; both were older, with a higher proportion of women.

The cohort with risk conditions and the cohort without the prevalence of diseases, polytherapy and CCI are different by design. Instead, the risk condition cohort showed a very similar distribution of clinical characteristics between contacted and non-contacted patients, with no clear selection between the two groups.

\section{Comparison}

Table 1 reports the estimates provided by the full logistic and Cox models; all predictors presented were included simultaneously in a unique model.

Mortality risk was significantly higher for older people $(\mathrm{HR}=7.53$; $\mathrm{OR}=10.98)$, patients who received more than 10 different treatments $(\mathrm{HR}=8.38 ; \mathrm{OR}=10.27)$, had chronic gastrointestinal diseases (inflammatory bowel disease (IBD) and cirrhosis) ( $\mathrm{HR}=32.69$; $\mathrm{OR}=66.57)$, chronic obstructive pulmonary disease (COPD, HR=34.64; OR=53.97), renal failure $(\mathrm{HR}=9.31$; $\mathrm{OR}=50.29)$, diabetes $(\mathrm{HR}=37.64 ; \mathrm{OR}=93.23)$, cardiovascular disease $(\mathrm{HR}=59.98 ; \quad \mathrm{OR}=374.8)$, neoplasm $(\mathrm{HR}=115.9 ; \mathrm{OR}=39.88)$, autoimmune diseases $(\mathrm{HR}=32.16$; $\mathrm{OR}=36.5)$, highest CCI score $(\mathrm{HR}=13.21 ; \mathrm{OR}=48.32)$ and influenza vaccination $(\mathrm{HR}=1.08 ; \mathrm{OR}=1.09)$. Female gender $(\mathrm{HR}=0.40$; $\mathrm{OR}=0.32)$ and pneumococcal vaccination ( $\mathrm{HR}=0.79 ; \mathrm{OR}=0.71$ ) were inversely associated with mortality risk. The C-index derived from the logistic model was very high, with a value of 0.93 , indicating an outstanding prediction of mortality.

The risk of having a positive RT-PCR test, based on nasal swabs, was significantly higher in older people $(\mathrm{HR}=2.37$; $\mathrm{OR}=2.32,90+$ years), patients who received more than 10 different treatments $(\mathrm{HR}=3.14 ; \mathrm{OR}=3.04)$, had $\mathrm{COPD}$ $(\mathrm{HR}=1.67 ; \mathrm{OR}=1.62)$, renal failure $(\mathrm{HR}=1.73 ; \mathrm{OR}=1.66)$, diabetes $(\mathrm{HR}=1.69 ; \mathrm{OR}=1.64)$, cardiovascular disease $(\mathrm{HR}=2.15 ; \mathrm{OR}=2.07)$, neoplasm $\quad(\mathrm{HR}=2.02 ; \mathrm{OR}=1.95)$, autoimmune diseases $(\mathrm{HR}=1.89$; $\mathrm{OR}=1.86)$ and highest CCI score $(\mathrm{HR}=2.17 ; \mathrm{OR}=2.03)$. The risk was inversely associated only with female gender $(\mathrm{OR}=0.53 ; \mathrm{HR}=0.54)$. The C-index derived from the logistic model was 0.70 , indicating an acceptable prediction of COVID-19 infection.

Risk of hospitalisation for COVID-19 was significantly higher for older people ( $\mathrm{HR}=1.66$; $\mathrm{OR}=1.61,90+$ years $)$, patients who received more than ten different treatments $(\mathrm{HR}=3.64 ; \mathrm{OR}=3.51)$, had $\mathrm{COPD} \quad(\mathrm{HR}=1.56$; $\mathrm{OR}=1.50)$, renal failure $(\mathrm{HR}=1.72 ; \mathrm{OR}=1.65)$, diabetes $(\mathrm{HR}=1.70 ; \mathrm{OR}=1.65)$, cardiovascular disease $(\mathrm{HR}=2.33$; $\mathrm{OR}=2.24)$, neoplasm $(\mathrm{HR}=2.06$; $\mathrm{OR}=1.99)$, autoimmune diseases $(\mathrm{HR}=1.90 ; \mathrm{OR}=1.87)$ and highest CCI score $(\mathrm{HR}=2.05 ; \mathrm{OR}=1.92)$. Hospitalisation risk was inversely associated with female gender $(\mathrm{HR}=0.43$; $\mathrm{OR}=0.44)$ and influenza vaccination $(\mathrm{HR}=0.89 ; \mathrm{OR}=0.89)$. The $\mathrm{C}$-index derived from the logistic model was 0.68 , indicating an acceptable prediction of hospitalisation for COVID-19 infection. 


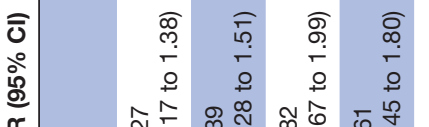
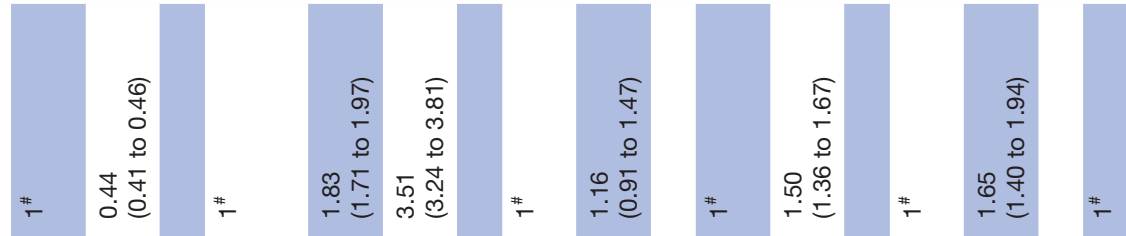

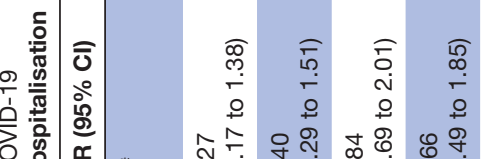

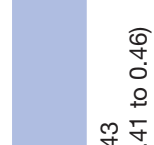

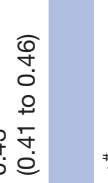

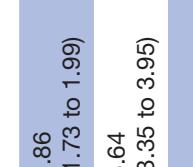

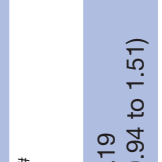

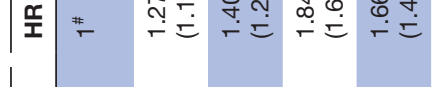

$\stackrel{0}{-}$

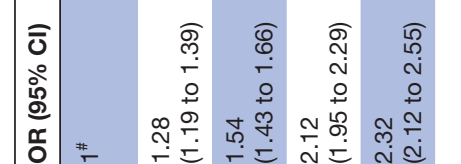

*

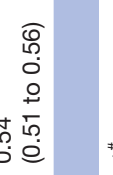

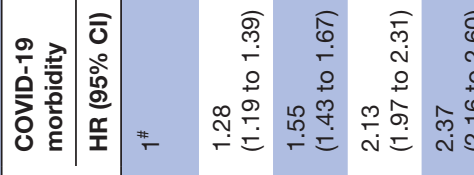

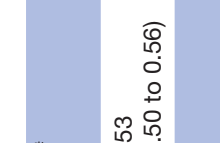

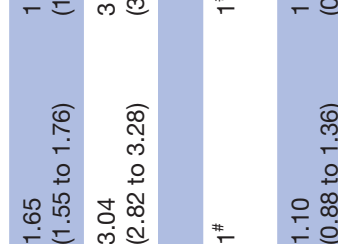

*

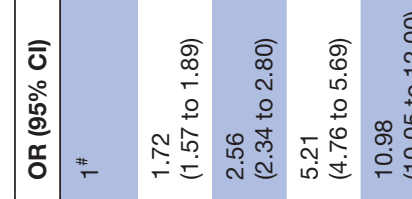

\#.

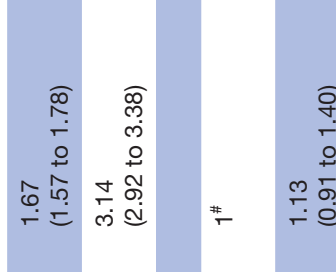

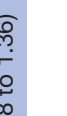
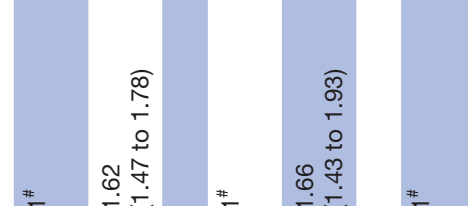

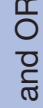

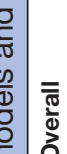

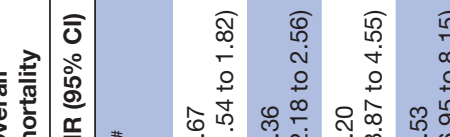

वे

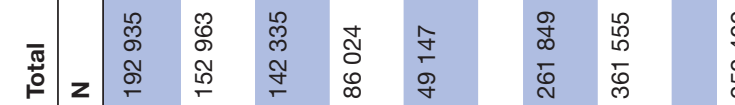

둔

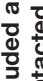

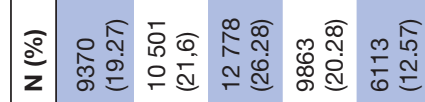

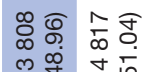

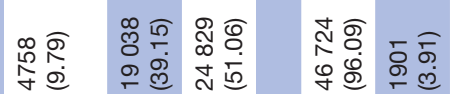

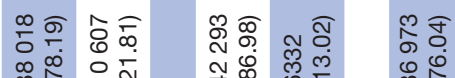

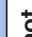

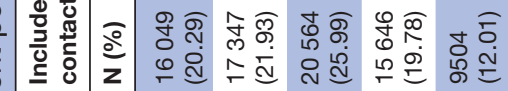

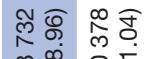

둔

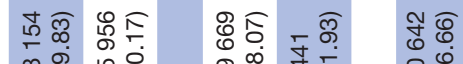

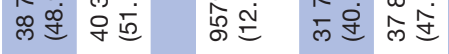

유워

ชำㄴำ

온
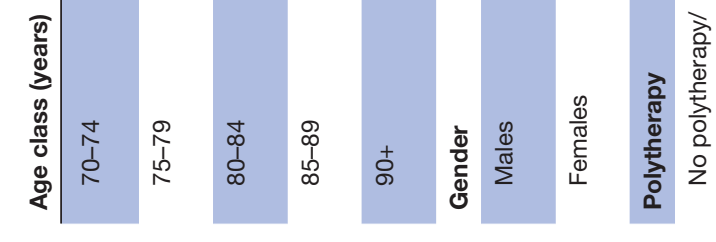
急过

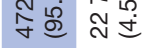




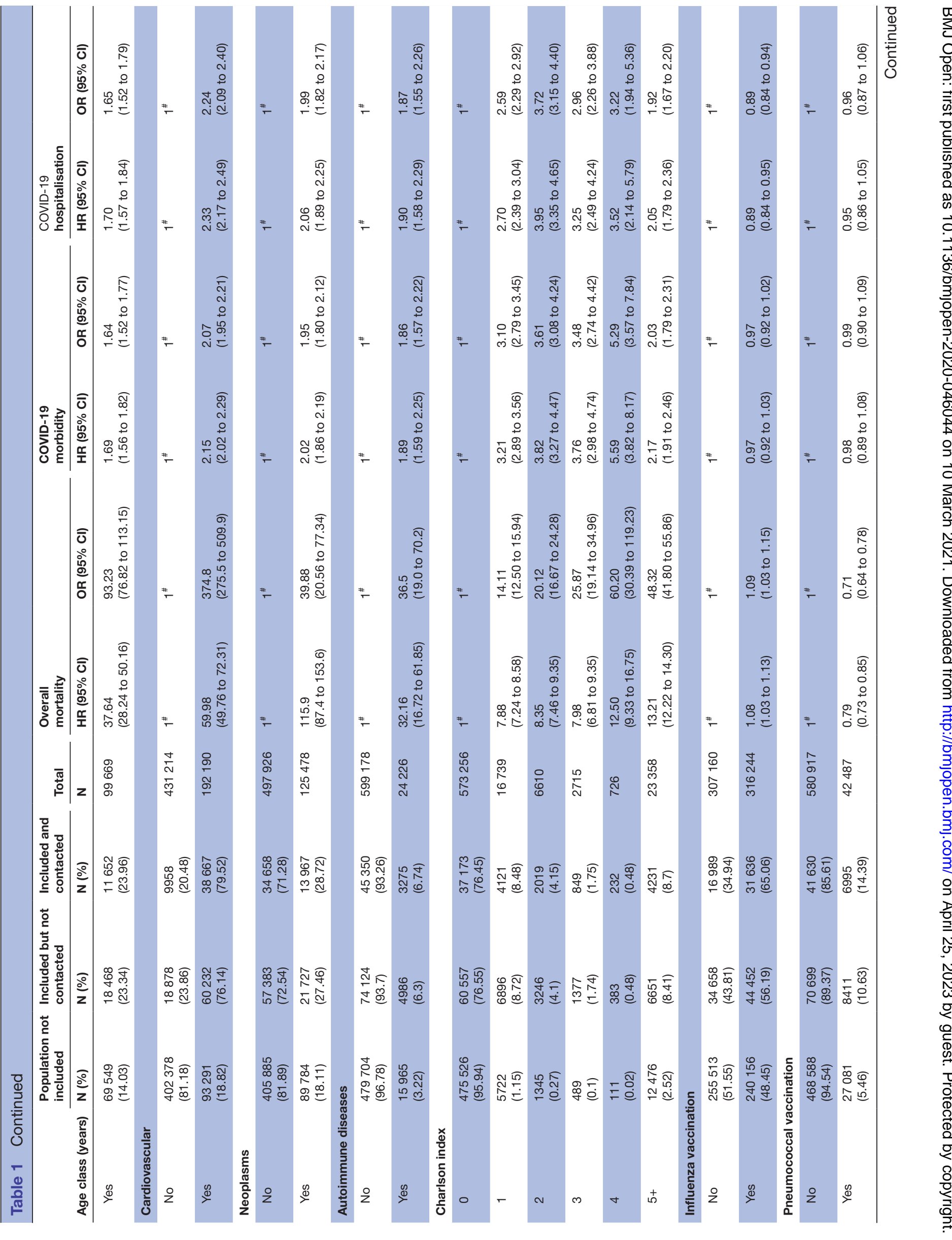


Outcomes

Overall mortality

Among the 623404 patients included in the populationbased study, 7831 died (1.26\%): overall mortality was $0.83 \%$ in the low-risk population (G1, 4125 events), $3.87 \%$ in the high-risk population not contacted by GPs (G2, 3065 events) and $1.32 \%$ in the high-risk population contacted by GPs (G3, 641 events) (table 2).

The high-risk population $(\mathrm{G} 2+\mathrm{G} 3)$ had a six to eight times increase in significant mortality risk, compared with the average-risk reference population $(\mathrm{HR}=6.24$; $\mathrm{OR}=8.30$ ), indicating good performance of the selection criteria identified. If the reference population was compared with the high-risk population subdivided based on GP intervention, the mortality risk for non-contacted patients was 8-11 times higher $(\mathrm{HR}=7.86$; $\mathrm{OR}=11.21)$; conversely, the contacted high-risk population had a threefold risk increase (HR=3.14; $\mathrm{OR}=3.89$ ). Comparing highrisk patients, contacted patients had half the mortality risk of non-contacted patients $(\mathrm{HR}=0.39 ; \mathrm{OR}=0.33)$.

\section{COVID-19 morbidity}

Out of 6166 patients with a positive RT-PCR test, based on nasal swabs, $0.79 \%$ were in the low-risk population (G1, 3.889 events), $2.46 \%$ in the high-risk population not contacted by GPs (G2, 1942 events) and $0.69 \%$ in the high-risk population contacted by GPs (G3, 335 events). The high-risk population showed a threefold risk increase, with respect to the reference population. When the reference population was compared with the two high-risk populations, the risks were very different: the non-contacted patients had a 1.4-3.7-fold risk increase, while contacted patients had the same risk as the reference population. When contacted and non-contacted patients were compared, the reduction in risk increase was $70 \%(\mathrm{HR}=0.29 ; \mathrm{OR}=0.30)$.

\section{COVID-19 hospitalisation}

A total of 5189 patients were hospitalised: $0.67 \%$ in the low-risk population (G1, 3343 events), $1.96 \%$ in the high-risk population not contacted by GPs (G2, 1548 events) and $0.61 \%$ in the high-risk population contacted by GPs (G3, 298 events). The high-risk population had a threefold risk increase compared with the reference population.

The risk of hospitalisation for COVID-19 did not differ between the reference and contacted population; instead, the non-contacted population had a threefold increase in risk. When contacted and non-contacted patients were compared, risk was shown to decrease by approximately $70 \%(\mathrm{HR}=0.32 ; \mathrm{OR}=0.34)$.

\section{DISCUSSION}

This study aimed to investigate whether patients with a specific predictive COVID-19 mortality profile show reduced mortality rates if they are under the care of GPs using a proactive and integrated model. The differences 
Table 2 Effect estimate for different sets of subjects, HRs from Cox models and ORs from logistic models and corresponding $95 \% \mathrm{Cls}$ for several outcomes

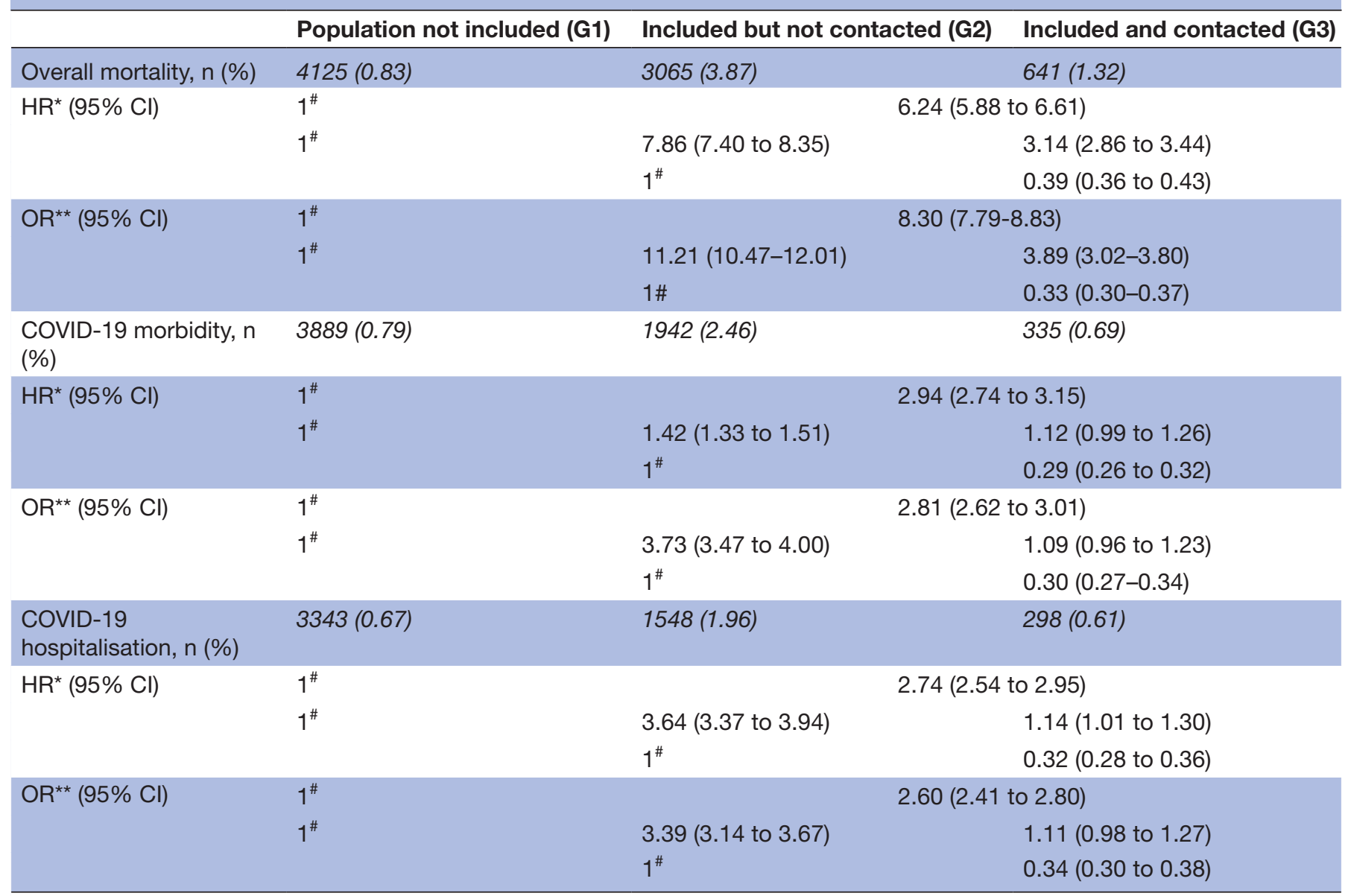

${ }^{\#}$ Referencecategory

between the active GP intervention group and the untreated group are striking and confirmed a functioning information system, particularly needed in future waves.

This study has two significant results. The first is the identification of a selection model of the population at risk based on where mortality, morbidity and hospitalisation events occurred. The second is that the group of patients who GPs engaged in multidisciplinary interaction showed a $50 \%$ reduction in mortality and in infection and hospitalisation risk compared with non-contacted patients.

The epidemiology of infectious diseases originated in 1854, during the London cholera epidemic, when the epidemiologist John Snow realised that it was possible to begin to understand the mechanisms of disease spread in the population, showing how great a role identification of new cases of infection in the population plays in maintaining population health. Since then, there have been many epidemics among various populations, and while a high price has been paid globally, there has also been an evolution in the methods for epidemic containment. The last major pandemic was the Spanish influenza epidemic over 100 years ago, which fell on a world devastated by a world war and a recession, and caused 50 million deaths in the absolute impossibility of any containment action.
According to WHO guidelines, ${ }^{17}$ the containment of an epidemic is based on the identification of epidemic clusters and their isolation, which allows, in particular situations, to identify and isolate their sources. Epidemiological investigation consists in the identification of a case and all potential close contacts and includes a targeted prescription of quarantine and information activity aimed at isolating the cluster. If the epidemic develops in a low-density or rural area, operators who conduct personal interviews can contact the case. For urban or densely inhabited areas, case identification is based on diagnostic testing. Therefore, cases are contacted a few days after the collection of biological material. Generally, this contact takes place in the form of telephone calls made by trained professionals who detect a range of structured information, including business contacts and identification of close contacts. However, if this modality can guarantee containment results for epidemic clusters of meningitis or legionella, dealing with a pandemic with these tools now becomes impractical due to the high number of cases to be contacted daily.

The epidemic produced several changes in primary care. GPs were on the front line in this epidemic, but as a consequence of the anti-COVID-19 measures, their activities were suspended with a first, direct effect: chronic care 
was frequently postponed. ${ }^{18}$ Patient-engaging strategies were developed by GPs via telephone or video consulting. Patient activation, or engaging patients in their own care, has been described as a potential strategy to improve chronic disease self-management. ${ }^{19}{ }^{20}$ However, this is conditioned by a pre-existing doctor-patient relationship.

The group followed by the GPs can be considered to have received true multidisciplinary treatment, represented by activation of social services; early evaluation of symptoms; dynamic monitoring of their condition by assessment of new symptom occurrence, changes in current therapy and indications for isolation even from family members; and the use of social distancing and other non-pharmaceutical practices. Only such a diverse range of treatments can explain such a high advantage.

\section{Limitations and strengths}

This study has several limitations, and the study design does not allow for causal inferences. Only the use of a randomised design can guarantee protection from confounding and justify causal inferences. However, not only is a randomisation procedure in a pandemic phase unethical, but it would be unacceptable for the population. The possibility of residual confounders cannot be eliminated. Although we controlled for important factors in the data analyses, this provides no guarantee for unbiased results. The definition of an algorithm for selection of the at-risk population, built at the beginning of the epidemic and based on limited data, is a critical issue. Moreover, the causes of death should be investigated in order to better understand how the GP intervention took effect in the high-risk population.

The two groups may differ in severity: table 1 shows a number of differences in terms of prevalence of chronic conditions between included patients. Contacted subjects mostly received concomitant therapies and have a slightly higher prevalence of some chronic diseases. If, indeed, GPs mainly contacted the most severe patients, it means that the potential efficacy of this approach is even greater than that observed.

The major strength of our work is represented by the population-based design including all people aged 70 years or older in the largest Italian metropolitan area, where the COVID-19 pandemic started in Italy.

\section{Conclusion}

When managing a pandemic crisis, it is extremely difficult to decide which strategy to adopt. If a government chooses suppression measures, there are substantial social and economic costs that will have long-term effects on the health and well-being of the population. If, however, a mitigation strategy is chosen and regular levels of activity are maintained, the healthcare system risks collapse, with a consequent dramatic increase in the number of deaths. A hypothetical new model could use suppression measures exclusively on compromised/the most vulnerable members of the population in order to control hospitalisation and mortality risk. Stringent measures of social distancing and individual protection could be introduced for the non-compromised population, along with economic and psychological support. This could represent a new overall strategy to protect the high-risk population and also mitigate the social and economic effect of the outbreak.

Innovative, comprehensive strategies need widespread social adhesion/cohesion and strong conviction in order not to create social ghettos, but to instead encourage the population to feel they can join in and help improve life. The possibility of early identification of risk groups that may require a compulsory extended quarantine, aimed at reducing adverse impacts, based on our results, shows promise as a viable path.

It is therefore crucial to foster collaboration among epidemiologists, ethicists, economists and sociologists, so that the social and economic consequences of control strategies are incorporated into the next generation of mathematical models used to guide public health decisions. Finally, it is necessary to reiterate that this intervention, which did not require any economic investment and which leveraged the skills of primary care services integrated with hospitals and the network of GPs, is one of the most successful actions, in terms of health gains, undertaken in public health during the COVID-19 epidemic.

Acknowledgements Agency for Health Protection of Milan COVID-19 Outbreak Working Group: the entire personnel involved in the departments, services and units of the Agency for Health Protection (ATS) of Milan. In particular, the Public Health Unit; Infectious Disease Surveillance Unit; Laboratory of Prevention; Occupational Health and Prevention Unit; and Epidemiology Unit. We would like to thank all general practitioners (GPs) that participated in the project, without whom this study would not have been possible.

Contributors Concept and design: AGR. Acquisition and interpretation of data: all authors. Drafting of the manuscript: AGR. Critical revision of the manuscript for important intellectual content: all authors. Statistical analysis: AGR. Administrative, technical or material support: all authors. AGR had full access to all of the data in the study and takes responsibility for the integrity of the data and the accuracy of the data analysis. AGR, the guarantor, accepts full responsibility for the work, had access to the data and controlled the decision to publish. The corresponding author attests that all listed authors meet authorship criteria and that no others meeting the criteria have been omitted.

Funding The authors have not declared a specific grant for this research from any funding agency in the public, commercial or not-for-profit sectors.

Competing interests All authors have completed the Competing Interest form (available on request from the corresponding author) and declare: no support from any organisation for the submitted work; no financial relationships with any organisations that might have an interest in the submitted work in the previous three years; and no other relationships or activities that could appear to have influenced the submitted work.

Patient and public involvement Patients and/or the public were involved in the design, or conduct, or reporting, or dissemination plans of this research. Refer to the Methods section for further details.

Patient consent for publication Not required.

Ethics approval Ethics approval and consent to participate were not required, as this is an observational study based on data routinely collected by the Agency for Health Protection (ATS) of Milan, a public body of the Regional Health Service of Lombardy. Among the institutional functions of the ATS, established by the Lombardy regional legislation (R.L. 23/2015), is the management of individual care in the regional social and healthcare system, the evaluation of services provided to patients residing in the area covered by the ATS and their health outcomes. This study is also ethically compliant with Italian law (Legislative Decree 101/2018) and the 'General Authorisation to Process Personal Data for Scientific Research 
Purposes' (clauses no. 8 and 9/2016, referred to in the Data Protection Authority action of 13/12/2018). Data were anonymised with a unique identifier in the different datasets before being used for the analyses.

Provenance and peer review Not commissioned; externally peer reviewed.

Data availability statement No data are available. The dataset from this study is held securely at the ATS of Milan, Epidemiology Unit. Data sharing agreements prohibit the ATS of Milan from making the dataset publicly available. The full dataset creation plan and underlying analytic code are available from the authors on request.

Open access This is an open access article distributed in accordance with the Creative Commons Attribution Non Commercial (CC BY-NC 4.0) license, which permits others to distribute, remix, adapt, build upon this work non-commercially, and license their derivative works on different terms, provided the original work is properly cited, appropriate credit is given, any changes made indicated, and the use is non-commercial. See: http://creativecommons.org/licenses/by-nc/4.0/.

\section{ORCID iD}

Antonio Giampiero Russo http://orcid.org/0000-0002-5681-5861

\section{REFERENCES}

1 EpiCentro. Sorveglianza integrata COVID-19: I principali dati nazionali, 2020. Available: https://www.epicentro.iss.it/coronavirus/ sars-cov-2-sorveglianza-dati

2 Lazzerini M, Putoto G. COVID-19 in Italy: momentous decisions and many uncertainties. Lancet Glob Health 2020;8:e641-2.

3 Sebastiani G, Massa M, Riboli E. Covid-19 epidemic in Italy: evolution, projections and impact of government measures. Eur J Epidemiol 2020;35:341-5.

4 Yang P, Qi J, Zhang S, et al. Feasibility study of mitigation and suppression strategies for controlling COVID-19 outbreaks in London and Wuhan. PLoS One 2020;15:e0236857.

5 Peng $\mathrm{T}$, Liu X, Ni H, et al. City lockdown and nationwide intensive community screening are effective in controlling the COVID-19 epidemic: analysis based on a modified Sir model. PLoS One 2020;15:e0238411.

6 Lau H, Khosrawipour V, Kocbach P, et al. The positive impact of lockdown in Wuhan on containing the COVID-19 outbreak in China. J Travel Med 2020;27:taaa037.

7 Flaxman S, Mishra S, Gandy A, et al. Estimating the effects of non-pharmaceutical interventions on COVID-19 in Europe. Nature 2020;584:257-61.
8 IATA. COVID-19 government public health mitigation measures, 2021. Available: https://www.iata.org/en/programs/covid-19resources-guidelines/covid-gov-mitigation/

9 Thompson RN, Hollingsworth TD, Isham V, et al. Key questions for modelling COVID-19 exit strategies. Proc Biol Sci 2020;287:20201405.

10 Stawicki SP, Jeanmonod R, Miller AC, et al. The 2019-2020 novel coronavirus (severe acute respiratory syndrome coronavirus 2) pandemic: a joint American College of academic international Medicine-World academic Council of emergency medicine multidisciplinary COVID-19 Working group consensus paper. J Glob Infect Dis 2020;12:47-93.

11 Zhou F, Yu T, Du R, et al. Clinical course and risk factors for mortality of adult inpatients with COVID-19 in Wuhan, China: a retrospective cohort study. Lancet 2020;395:1054-62.

12 Guan W-J, Ni Z-Y, Hu Y, et al. Clinical characteristics of coronavirus disease 2019 in China. N Engl J Med 2020;382:1708-20.

13 Vahidy FS, Nicolas JC, Meeks JR, et al. Racial and ethnic disparities in SARS-CoV-2 pandemic: analysis of a COVID-19 observational Registry for a diverse us metropolitan population. BMJ Open 2020;10:e039849.

14 Richardson S, Hirsch JS, Narasimhan M, et al. Presenting characteristics, comorbidities, and outcomes among 5700 patients hospitalized with COVID-19 in the new York City area. JAMA 2020;323:2052-9.

15 Charlson M, Szatrowski TP, Peterson J, et al. Validation of a combined comorbidity index. J Clin Epidemiol 1994;47:1245-51.

16 Harrell FE, Lee KL, Mark DB. Multivariable prognostic models: issues in developing models, evaluating assumptions and adequacy, and measuring and reducing errors. Stat Med 1996;15:361-87.

17 WHO. Country \& technical guidance - Coronavirus disease (COVID-19), 2021. Available: https://www.who.int/emergencies/ diseases/novel-coronavirus-2019/technical-guidance

18 Morreel S, Philips H, Verhoeven V. Organisation and characteristics of out-of-hours primary care during a COVID-19 outbreak: a real-time observational study. PLoS One 2020;15:e0237629.

19 Blackberry ID, Furler JS, Best JD, et al. Effectiveness of general practice based, practice nurse led telephone coaching on glycaemic control of type 2 diabetes: the patient engagement and coaching for health (peach) pragmatic cluster randomised controlled trial. BMJ 2013;347:f5272.

20 Oksman E, Linna M, Hörhammer I, et al. Cost-Effectiveness analysis for a tele-based health coaching program for chronic disease in primary care. BMC Health Serv Res 2017;17:138. 